\title{
Constitution et lois fondamentales chez Rousseau
}

\author{
Constituição e leis fundamentais em Rousseau
}

\section{Gabrielle Radica*}

Université de Picardie-Jules Verne, Amiens, France

\section{Resume}

Pour examiner la pertinence du lien que ses lecteurs ont établi entre la pensée de Rousseau d'une part, et la théorie des droits de l'homme d'autre part, et dans la mesure où les occurrences de l'expression de « droits de l'homme » sont anecdotiques dans l'œuvre du philosophe genevois, une question préjudicielle peut être posée, celle du constitutionnalisme de Rousseau. L'auteur conceptualise en effet la notion de constitution d'une part, celle de lois fondamentales de l'autre. Il est vrai que l'absolue priorité de la volonté générale et de la souveraineté populaire interdit qu'aucun texte positif puisse peser sur la vie politique et enchaîner la liberté collective, mais il n'en reste pas moins que dans les textes où Rousseau se penche sur la question de la durée de vie des corps politiques, il reconnaît la nécessité de protéger certaines lois contre l'instabilité législative, et accorde à ces dernières le nom de « lois fondamentales », en exigeant pour elles dans les Considérations sur le gouvernement de Pologne des conditions de modification

* GR : Doutora em Filosofia, e-mail : gabrielle.radica@u-picardie.fr 
plus solennelles et plus strictes que pour les autres lois. C'est bien reconnaître une hiérarchie des normes, que la thèse volontariste de la souveraineté populaire interdisait.

Mots-clés : Rousseau. Droits de I'homme. Lois fondamentales. Constitutionnalisme. Souveraineté populaire.

\section{Resumo}

Para examinar a pertinência da ligação que seus leitores estaleceram entre o pensamento de Rousseau, de uma parte, e a teoria dos direitos humanos, de outra, e na medida em que as ocorrências da expressão " direitos do homem » são anedóticas na obra do filósofo genovês, uma questão preliminar pode ser colocada, aquela do constitucionalismo de Rousseau. De fato, o autor conceitualiza, de um lado, a noção de constituição, como as leis fundamentais do outro. É verdade que que a absoluta prioridade da vontade geral e da soberania popular interdita que algum texto positivo possa pesar sobre a vida política e aprisionar a liberdade coletiva, mas isso não consta a não ser nos textos nos quais Rousseau examina a questão da duração de vida dos corpos políticos, ele reconhece a necessidade de proteger certas leis contra a instabilidade legislativa e concebe para esses últimos o nome de «leis fundamentais ", exigindo para elas, nas "Considerações sobre o governo da Polônia, " condições de modificação mais solenes e mais estritas do que para as outras leis. É bom reconhecer uma hierarquia das normas, algo que a tese voluntarista da soberania popular interditou.

Palavras-chaves : Rousseau. Direitos do homem. Leis fundamentais. Constitucionalismo. soberania popular.

\section{Introduction}

Dans L'invention des droits de l'homme, Lynn Hunt (2013, p. 23) affirme que la notion de " droits de l'homme » a été historiquement associée à Rousseau, par exemple par Burke, dans ses Réflexions sur la révolution de France [1790], mais même aussi avant la révolution, juste après la parution du Contrat social (HUNT, 2013, p. 30-31). L'auteure 
est singulièrement vague à propos de Rousseau, puisqu'elle lui prête une confusion qui n'est que celle de ses propres thèses ou celle des propos des lecteurs de Rousseau : « ce dernier [Rousseau] n'en donne cependant aucune définition [s.e. des droits de l'homme] et parle indistinctement de «droits de l'humanité », de "droits du citoyen » et de « droits de la souveraineté » ». Et pour cause. Les droits de l'homme ne sont pas un concept rousseauiste. On ne saurait mieux que Lynn Hunt mélanger ce qui doit rester distinct, et faire pire justice à la rigueur d'un auteur. Au lieu de douter raisonnablement que des expression aussi distinctes puissent avoir la moindre chance de renvoyer à un concept unique de «droits de l'homme », que l'auteure impose au demeurant faussement à Rousseau comme étant le sien ${ }^{1}$, elle préfère penser qu'il a introduit le concept, puis ne l'a nommé que par des expressions autres, et l'a par ailleurs très mal pensé. On ne saurait plus mal penser non plus ni étudier un concept « dans » un auteur indépendamment de tout ce qu'il écrit. La leçon intéressante de cette analyse d'histoire des représentations réside néanmoins dans le récit que fait Lynn Hunt de l'association que ses lecteurs ont réalisée entre l'œuvre de Rousseau et la problématique des droits de l'homme, notamment durant la Révolution.

Suivant une méthode plus prudente pour savoir en quoi Rousseau peut éclairer notre compréhension des droits de l'homme, alors que cette expression ne se trouve pas dans l'œuvre, nous traiterons des notions travaillées par Rousseau, et dont la compréhension est utile pour celle des droits de l'homme, car elles rencontrent des problèmes communs: il s'agit de la constitution et des lois fondamentales. De même que les droits de l'homme, les lois fondamentales et la constitution sont des normes qui peuvent au besoin opposer leur stabilité et leur caractère supérieur aux aleas de la vie politique ordinaire, qui constituent une barrière pour le législateur lui-même. On est en droit de se demander si Rousseau est un penseur de la constitution. Le terme

1 «Droits de I'homme » se retrouve en effet une fois dans le Contrat social, au moment d'étudier les religions, au livre IV, chapitre XII, mais il n'est qu'une partie de l'expression qui prise dans son ensemble en modifie le sens : « devoirs et droits de I'homme». 
est présent dans ses textes : dans le Contrat social, en II, 12, il affirme que les mœurs font « la véritable constitution de l'Etat » ${ }^{2}$, et en III, 11, que « La constitution de l'homme est l'ouvrage de la nature, celle de l'Etat est l'ouvrage de l'art ${ }^{3}$ et il est précisé ensuite que la constitution de l'Etat est un corps de lois fixé une fois par le peuple qui lui donne sa sanction 4 . Rappelons que Rousseau a écrit un Projet de constitution pour la Corse.

Pourtant la notion de constitution est partiellement vidée du sens que nous lui attribuons à partir de l'âge moderne : si Rousseau reconnaît dans l'Etat une constitution au sens d'un corps de lois fixées par une volonté humaine, et s'il la compare selon une métaphore organique à la conformation, ou physionomie d'un Etat, qui naît, se corrompt et meurt ${ }^{5}$, ceci le rattache plus à ce qu'Aristote désigne dans les Politiques comme une constitution ${ }^{6}$, politeia, qu'à ce que l'on appellera par la suite la tradition constitutionnaliste ${ }^{7}, c^{\prime}$ est-à-dire une pensée des limites du pouvoir qui viendrait, soit du jeu interne de ses organes, comme c'est le cas du régime anglais décrit par Montesquieu en Esprit des lois, XI, 6, soit d'une règle ou d'un texte écrit auquel le pouvoir se soumettrait parce que l'obligation pèserait sur lui de le faire, comme c'est le cas des textes constitutionnels genevois qui organisent les droits respectifs du souverain et du conseil (voir l'Edit de Médiation de 1538). Rousseau est parfaitement au courant de ce que signifie une constitution en ce sens, mais sa conception de la souveraineté populaire lui interdit d'accepter dans l'Etat un texte législatif qui enchaînerait la volonté générale: «Ce n'est point par les lois que 1'Etat subsiste, c'est par le pouvoir législatif. La loi d'hier n'oblige pas aujourd'hui $»^{8}$.

En outre, Rousseau intervient au sein de différentes réinterprétations et redéfinitions de la notion de « lois fondamentales »-lesquelles

\footnotetext{
CFuvres Complètes, éd. Raymond et Gagnebin, 5 vol., Paris, La Pléiade-Gallimard, tome III, p. 394 (noté désormais OC III).

Contrat social, III, XI, OC III, p. 424.

Contrat social, III, XIII, OC III, p. 426.

Contrat social, III, XI, OC III, p. 424-425.

Et qui continue de travailler dans les textes de Machiavel ou de Spinoza par exemple.

Sur la tradition constitutionnaliste, voir par exemple Keohane, 1980.

Contrat social, III, XI, OC III, p. 424.
} 
désignent historiquement des règles de dévolution du pouvoir royal - qui ont été effectuées aux XVIIe et XVIIIe siècles. Les lois fondamentales nous intéressent ici autant que l'idée de constitution, parce que, comme la constitution, elles constituent des règles capables de limiter le pouvoir de l'extérieur.

À côté de l'interprétation coutumière, longtemps prédominante en France, des « lois fondamentales », s'est développée une autre explication des lois fondamentales dans les auteurs du droit naturel. C'est une réappropriation rationnelle de lois positives qui est alors effectuée. Les lois fondamentales sont chez Samuel Pufendorf des promesses particulières faites par le roi, qui déterminent les modalités que prendra la formulation de sa volonté, et l'obligent même parfois, sous peine de perdre l'obéissance de ses sujets : l'auteur est clair sur ce point, il s'agit bien de "limitations de la souveraineté ${ }^{9}$. On retrouve des thèses proches chez Jean-Jacques Burlamaqui :

Mais quoiqu'il soit absolument nécessaire qu'il y ait dans l'Etat une puissance souveraine et dépendante, il y a cependant quelque différence, surtout dans les monarchies et les aristocraties, dans la manière dont ceux à qui ce pouvoir est confié l'exercent. Dans quelques États, le prince gouverne comme il le juge à propos ; dans d'autres, il est obligé de suivre certaines règles fixes et constantes, dont il ne saurait s'écarter : c'est ce que j'appelle les modifications de la souveraineté, et c'est de là que naît la distinction de la souveraineté absolue et de la souveraineté limitée.

Le souverain est pour ces auteurs au-dessus de toute loi humaine, mais il y a une différence entre les lois qui sont décidées et

9 Droit de la nature et des gens (désormais noté DNG), 1987 (t.II, I.VII, ch. 6, §IX, p. 290-319), p. 298 : « Cependant, comme une seule personne peut se tromper aisément dans l'examen de ce qui concerne le bien public; et que d'ailleurs tous les princes n'ont pas assez de vertu et de courage pour modérer leurs passions, quand ils se voient tout permis : plusieurs peuples ont jugé à propos de mettre des bornes au pouvoir de leurs souverains, et de leur prescrire la manière dont ils doivent gouverner ; surtout depuis qu'on a remarqué, que le génie de chaque nation, et la constitution de chaque Etat demande certaines lois, et certaine manière de gouvernement. Par cette limitation de la souveraineté, on ne fait aucun tort aux princes qui sont élevés sur le trône en vertu d'un libre consentement du peuple. Car s'ils ne pouvaient se résoudre à n'avoir qu'une autorité bornée, il ne tenait qu'à eux de refuser la couronne. La promesse solennelle quíls font en l'acceptant ne leur permet pas non plus de travailler ensuite à renverser les lois fondamentales du royaume et à se rendre absolus, ou par une force ouverte, ou, par de sourdes pratiques. ». 
autorisées par le souverain, auxquelles il ne peut être soumis, et les lois qui encadrent l'exercice du pouvoir souverain lui-même et auxquelles en revanche il n'est pas contradictoire qu'il soit soumis. «Ces règlements, qui restreignent l'autorité souveraine, qui lui donnent des bornes, sont appelés lois fondamentales de l'Etat (BURLAMAQUI, 1983, p. 60-88)».

Montesquieu a fourni de son côté une interprétation de cette notion très originale, dépouillée de tout volontarisme et telle que les lois fondamentales désignent les lois de fonctionnement nécessaires, essentielles de tel ou tel régime. Ces lois sont placées, du point de vue de leur importance, entre la nature et le ressort du gouvernement ${ }^{10}$. Elles permettent à l'auteur d'expliciter les exigences de la nature du gouvernement, et de tout type de gouvernement : elles ne concernent donc plus seulement le gouvernement monarchique, et par ailleurs, elles ne sont ni des coutumes, ni des productions de telle ou telle volonté, car elles sont des lois impersonnelles de rapports entre les choses.

Or Rousseau est en retrait vis-à-vis de ces innovations théoriques, et il restitue aux lois fondamentales la signification classique et historique, de règles devant encadrer et limiter l'exercice du pouvoir et de la souveraineté. Mais il ne garde cette définition que pour mieux rejeter la pertinence et la légitimité de telles lois fondamentales, et ce en invoquant, comme il le fait au sujet de la constitution, la souveraineté populaire. A propos des lois politiques, qui règlent le rapport du souverain à lui-même, qui organisent l'Etat, il affirme ainsi :

Les lois qui règlent ce rapport portent le nom de lois politiques, et s'appellent aussi lois fondamentales, non sans quelque raison si ces lois sont sages. Car s'il n'y a dans chaque Etat qu'une bonne manière de l'ordonner, le peuple qui l'a trouvée doit s'y tenir ; mais si l'ordre établi est mauvais, pourquoi prendrait-on pour fondamentales des lois qui l'empêchent d'être bon ? ${ }^{11}$

10 C'est Jean Ehrard (2005-5, p. 267-278), qui a proposé cette interprétation lumineuse.

11 Contrat social, II, XII, OC III, p. 393-394. 
Rousseau rejette toute forme de contrainte extérieure au pouvoir populaire et de là, toute forme de constitutionnalisme, y compris dans la forme traditionnelle des lois fondamentales.

On peut comprendre alors pourquoi Rousseau est invoqué au invoqué pendant la Révolution pour fonder une tradition démocratique contre laquelle se précise une conception libérale du pouvoir. La première s'exprime par exemple dans la Constitution du 24 juin 1793, dans l'article 28, qui stipule qu' «Un peuple a toujours le droit de revoir, de réformer et de changer sa constitution. Une génération ne peut assujettir les générations futures ». Entre ce texte constitutionnel et Rousseau a peut-être joué la médiation du texte de Thomas Paine qui dans Les droits de l'homme, en 1791, accepte l'idée de constitution tout en rappelant la prééminence du peuple dans l'assemblée :

Je défends les droits des vivants, et je m'efforce d'empêcher qu'ils ne soient aliénés, altérés ou diminués par l'autorité usurpée des morts ; et Monsieur Burke prend le parti de l'autorité des morts contre les droits et contre la liberté des vivants ${ }^{12}$.

Le refus de limites écrites, donc pérennes, et extérieures au pouvoir souverain, a une histoire, qui est liée à l'histoire de la notion de pouvoir constituant ${ }^{13}$. En fonction de l'intensité de sa présence effective sur la scène politique, ce pouvoir constituant du peuple peut quasiment empêcher toute stabilité de la constitution s'il lui est supérieur et si celle-ci attend d'être validée et reconduite par lui ${ }^{14}$.

La tradition libérale quant à elle rassemble des auteurs marqués par l'épisode de la Convention, comme Siéyès, Constant, qui craignent

12 Les droits de l'homme, éd. Claude Mouchard, Paris, Belin, 1987, p. 75. Voir aussi par exemple p. 74 : « II n'y eut, il n'y aura jamais, il est même impossible qu'il existe dans aucun temps ou dans aucun pays un parlement qui ait le droit de lier la postérité jusqu'à la consommation des siècles, ou de commander de quelle manière le monde doit être gouverné, et par qui il sera gouverné jusqu'à l'éternité ».

13 Jean-Fabien Spitz en reconstitue la généalogie dans des auteurs comme Lawson, Rutherford puis Locke, dans John Locke et les fondements de la liberté moderne (2001).

14 Si l'on parvient en revanche à formuler l'idée que le pouvoir constituant est parfois seulement virtuel par exemple, on redonne une autonomie propre au gouvernement vis-à-vis du pouvoir constituant. Sur tout cela, voir les analyses très éclairantes de Spitz, dans l'ouvrage mentionné ci-dessus. 
les débordements des assemblées populaires ${ }^{15}$. Le "libéralisme » constitutionnel regroupe ainsi ceux qui conçoivent la république plutôt comme un Etat de droit, doté de différents garde fous, de limites qui encadrent la vie politique (règne de la loi, liberté de la presse, laïcité, décentralisation, etc.), au sein desquels une constitution écrite joue un rôle central. Rousseau est certes moins proche de cette tradition que des positions de Paine et des courants démocratiques cités plus haut. Néanmoins ses thèses sont complexes et on cherchera ici quelles concessions l'auteur accorde malgré tout à la position constitutionnaliste, notamment dans les textes de philosophie politique " appliquée » (Projets pour la Corse et la Pologne, Lettres écrites de la Montagne).

Rousseau discerne deux composantes essentielles dans la notion de loi fondamentale. D'une part les lois fondamentales, en ce qu'elles limitent la souveraineté, sont critiquées dans le Contrat social [1762] en tant que limites opposées à la souveraineté populaire, mais elles sont acceptées dès qu'il s'agit de soumettre le gouvernement au souverain. D'autre part, dès le Discours sur l'économie politique [1755], mais aussi dans le Contrat social, et jusque dans les Considérations sur le gouvernement de Pologne [1772], les lois fondamentales sont examinées en tant qu'elles participent du maintien de la constitution de l'Etat, de sa durée et de sa stabilité. Il y a donc une tension au sujet des lois fondamentales et de la constitution, entre volontarisme et position souverainiste d'une part, et considération de la durée de l'Etat de l'autre. Parce que le traitement du problème de la stabilité est conditionné par celui de la souveraineté, il convient d'étudier d'abord le premier point, à savoir le refus rousseauiste de toute hiérarchie des lois, au nom du principe souverainiste (1). Toutefois Rousseau accepte d'appliquer le terme de « constitution » au corps politique (2), ainsi qu'au gouvernement (3). Mais c'est l'abandon du point de vue contractualiste et la considération de la durée des corps politiques qui révèlent une vraie difficulté de ces revendications souverainistes, difficulté que l'auteur reconnaît (4). Une des solutions qu'il y donnera plus tardivement est le choix de

15 On pourra se reporter à la mise en contexte de ces auteurs dans la longue histoire du libéralisme qu'effectue Catherine Audard dans Qu'est-ce que le libéralisme (2009). 
différencier les lois fondamentales et les lois non fondamentales (notamment dans le projet sur la Pologne) (5).

\section{Le souverainisme contre les lois fondamentales et la constitution}

Le refus des lois fondamentales et de la problématique constitutionnaliste vient de l'affirmation d'une position souverainiste forte, que Rousseau retravaille chez ses prédécesseurs puisqu'il ne la reprend que dans la mesure où elle est favorable au peuple. Cette position souverainiste éclate dans le Contrat social dont elle est une thèse directrice ${ }^{16}$, notamment au livre II.

Refusant la dissociation établie par Pufendorf entre celui qui possède la souveraineté et celui qui l'exerce ${ }^{17}$, parce qu'elle est trop favorable aux monarques, Rousseau critique également les thèses de Pufendorf et de Grotius, sur la possibilité que le souverain gouverne

16 Pour ce qui est du second Discours, on trouve une mention singulière des lois fondamentales. Rousseau y admet en effet de façon concessive et comparative (pour souligner l'iniquité de la tyrannie) que le souverain est « sujet aux lois de son Etat ». Mais ce qu'il appelle souverain dans ce texte est en fait ce qu'il appellera gouvernement dans le Contrat social, à savoir le monarque. À l'appui de cette affirmation, il cite le Traité des droits de la reine très chrétienne sur divers États de la monarchie d'Espagne (1667), écrit de circonstance anonyme commandé par Louis XIV, où sont invoquées effectivement des lois fondamentales (voir André Lemaire, Les lois fondamentales de la monarchie française d'après les théoriciens de l'ancien régime, Paris, 1907, reprint Genève, Slatkine-Megariotis, 1975, p. 189, qui le cite : « La loi fondamentale, ayant formé une liaison éternelle et réciproque entre le Prince et ses descendants d'une part, et les sujets et leurs descendants de l'autre, par une espèce de contrat qui destine le souverain à régner, et les peuples à obéir, nulle des parties ne peut seule et quand il lui plaît se déliurer d'un engagement si solennel dans lequel ils se sont donnés les uns aux autres pour s'entraider mutuellement [...]. Les rois par un attribut même de leur souveraineté, et par la propre excellence et perfection de leur sacré caractère, sont dans une heureuse impuissance de détruire les lois de leur Etat ni renverser au préjudice du droit public les coutumes particulières de leurs provinces. Ce n'est ni imperfection ni faiblesse dans une autorité suprême, que de se soumettre à la foi de ses promesses, ou à la justice de ses lois »). Rousseau cite ce texte sans en donner le titre : "un écrit célèbre publié en 1667, au nom et par les ordres de Louis XIV », voir Second Discours, OCIII, p. 183. La phrase que Rousseau extrait du Traité est la suivante : "Qu'on ne dise donc point que le souverain ne soit pas sujet aux lois de son Etat, puisque la proposition contraire est une vérité du droit des gens que la flatterie a quelquefois attaquée, mais que les bons princes ont toujours défendue comme une divinitétutélaire de leurs États. Combien est-il plus légitime de dire avec le sage Platon, que la parfaite félicité d'un royaume est qu'un roi soit obéi de ses sujets, que le prince obéisse à la loi, et que la loi soit droite et toujours dirigée au bien public ». Mais que l'utilisation par Rousseau de ce texte soit naïve ou ironique, elle revient au même : l'engagement civil ne peut concerner une seule des parties.

17 Contrat social, II, I, OC III, p. 368-369.

Rev. Filos., Aurora, Curitiba, v. 28, n. 43, p. 19-38, jan./abr. 2016 
pour d'autres raisons que l'utilité du peuple, et sur la possibilité que le peuple se fasse esclave d'un prince.

Mais une fois affirmée l'inaliénabilité de la souveraineté populaire, Rousseau reprend à son compte certains des attributs de la souveraineté telle qu'elle a théorisée par Pufendorf, notamment ceux qui sont indépendants de l'idée d'une délégation. C'est le fait que le souverain n'a pas de loi au-dessus de lui qui intéresse particulièrement Rousseau, notamment le fait que le souverain ne peut être soumis à aucune loi, même pas celle qu'il aurait énoncée lui-même. Afin de défendre les prérogatives des souverains, Pufendorf affirme en effet que « l'on ne saurait se prescrire à soi-même aucune loi proprement ainsi nommée, c'est-àdire, à laquelle on soit tenu d'obéir comme venant d'un supérieur ${ }^{18}$ et s'il arrive que le souverain qui par définition n'est soumis qu'à lui-même respecte ses propres lois, c'est par honnêteté, ou pour donner l'exemple.

Or, Barbeyrac son traducteur s'est penché sur l'application de cette maxime aux cas où le peuple est souverain, à savoir dans les démocraties. Ce faisant, Barbeyrac prépare certainement l'application qu'en fera Rousseau à un peuple dont la souveraineté est de surcroît inaliénable. Dans sa note au texte de Pufendorf, Barbeyrac affirme ainsi qu'un peuple libre peut se donner des lois, et que chaque citoyen y obéira, parce qu'en tant que particulier, le citoyen est soumis au corps collectif du peuple qui lui est supérieur, mais « le peuple entier ne s'engage à rien, puisqu'il ne saurait être supérieur de lui-même ${ }^{19}$.

Or la thèse de Barbeyrac a des effets encore plus surprenants une fois reformulée chez Rousseau car elle aboutit à l'impossibilité absolue de toute sorte de loi fondamentale :

Je suppose ici ce que je crois avoir démontré, savoir qu'il n'y a dans l'Etat aucune loi fondamentale qui ne se puisse révoquer, non pas même le pacte social; car si tous les citoyens s'assemblaient pour rompre ce pacte d'un commun accord, on ne peut douter qu'il ne fût très légitimement rompu. Grotius pense même que chacun peut renoncer à l'Etat dont il est membre, et reprendre sa liberté naturelle et ses biens en sortant du

18 DNG, t. 2, livre VII, ch. VI, § III, p. 291-292.

19 Note (4), ibid. 
pays. Or il serait absurde que tous les citoyens réunis ne pussent pas ce que peut séparément chacun d'eux. ${ }^{20}$

\section{La seule loi fondamentale, c'est le pacte social}

Mais ceci n'épuise pas les usages du terme de constitution et de loi fondamentale que l'on repère dans l'œuvre de Rousseau. En effet, quand l'auteur reprend le vocabulaire constitutionnaliste, ou des expressions comme celle de " loi fondamentale ", c'est tantôt pour rejeter la théorie qui y correspond, tantôt pour absorber ce vocabulaire dans son propre cadre conceptuel. Le peuple souverain ne peut se voir imposer de lois qui seraient au dessus de lui ; il est toujours au dessus d'elles $^{21}$. Si l'on veut malgré tout employer le terme de constitution, on devra dire que seul le contrat social peut être appelé la constitution du peuple. De fait c'est en effectuant le contrat social que le corps moral et collectif du peuple est produit par l'acte d'association ${ }^{22}$. Quant à la loi fondamentale, l'idée est formulée de manière on ne peut plus nette dans l'Emile : « il n'y a ni ne peut y avoir d'autre loi fondamentale proprement dite que le seul pacte social $»^{23}$.

Récapitulons : il n'y a pas de place dans le Contrat social pour une quelconque constitution ou loi fondamentale entendue comme loi qui se situerait au-dessus du souverain populaire. En revanche, on peut à la rigueur appeler constitution le contrat social lui-même, puisqu'effectivement c'est le premier acte du peuple qui s'est « constitué » comme peuple en le passant. Cependant, Rousseau admet aussi de recourir au terme de constitution, sans plus cette fois être gêné par la connotation de subordination qu'implique cet usage, quand il parle des rapports du souverain au gouvernement. Le gouvernement, corps à part dans l'Etat, reçoit bien du souverain auquel il est subordonné, une constitution qu'il doit respecter et qu'il ne peut modifier lui-même.

\footnotetext{
20 Contrat social, III, 18, OC III, p. 436.

21 Contrat social, III, XI, OC III, p. 424-425.

22 Voir Contrat social, I, VI, OC III, p. 361.

23 Emile, OC IV, p. 840.
} 


\section{Le gouvernement est soumis au peuple dont il reçoit sa constitution}

Dans le Contrat social, une fois la distinction établie entre souverain et gouvernement, à partir du livre III, le gouvernement doit à son tour être constitué par le souverain :

Cependant, pour que le corps du gouvernement ait une existence, une vie réelle qui le distingue du corps de l'Etat, pour que tous ses membres puissent agir de concert et répondre à la fin pour laquelle il est institué, il lui faut un moi particulier, une sensibilité commune à ses membres, une force, une volonté propre qui tende à sa conservation ${ }^{24}$.

Parler de constitution à propos du gouvernement ne pose plus de problème de légitimité puisque, pour reprendre les termes ultérieurs de Sieyès ${ }^{25}$, le gouvernement est un pouvoir commis qui ne peut rétroagir sur le pouvoir commettant.

Cette existence particulière suppose des assemblées, des conseils, un pouvoir de délibérer, de résoudre, des droits, des titres, des privilèges qui appartiennent au prince exclusivement et qui rendent la condition du magistrat plus honorable à proportion qu'elle est plus pénible. ${ }^{26}$

Et Rousseau utilise alors le terme de " constitution », au moment d'affirmer que la complexité de la tâche du législateur consiste à ajuster dans la constitution du tout, dans la « constitution générale », la constitution propre du gouvernement qui « ne doit pas affaiblir » la première. Jean Terrel a montré dans Les théories du pacte social (2001) que le contractualisme, traditionnellement associé à l'idée de limites du pouvoir et de devoirs ou de promesses du souverain envers le peuple, perd cette fonction limitative chez Rousseau, puisque le souverain est le peuple en personne :

24 Contrat social, III, I, OC III, p. 399.

25 Qu'est-ce que le tiers-état?, in Écrits politiques, éd. Roberto Zapperi, Gordon and Breach, 1994, ch. V, p. 169 : « Aucune sorte de pouvoir délégué ne peut rien changer aux conditions de sa délégation. C'est ainsi et non autrement que les lois constitutionnelles sont fondamentales. »

26 Ibid. 
Ainsi, ceux qui prétendent que l'acte par lequel un peuple se soumet à ses chefs n'est point un contrat ont grande raison. Ce n'est absolument qu'une commission, un emploi dans lequel, simples officiers du souverain, ils exercent en son nom le pouvoir dont il les a faits dépositaires, et qu'il peut limiter, modifier et reprendre quand il lui plaît, l'aliénation d'un tel droit étant incompatible avec la nature du corps social, et contraire au but de l'association ${ }^{27}$.

En revanche, le gouvernement est soumis au souverain, d'une façon unilatérale. Un pacte lui donnerait un droit de regard que Rousseau ne souhaite pas lui laisser. Et si un pacte l'unissait au peuple, qui arbitrerait de son respect en cas de conflit? Faute de juge légitime en ces conflits, on risquerait de retourner à l'état de nature qu'on viendrait de quitter.

Ainsi, le gouvernement est le pouvoir constitué qui reçoit ses limites et sa forme du souverain : en ce sens, il y a bien des limites au pouvoir gouvernemental, mais celles-ci jouent à rebours du sens qu'attendent les libéraux, puisqu'elles jouent en faveur du peuple assemblé et ne sont pas instaurées pour le modérer.

\section{L'Etat doit durer et maintenir certaines lois}

En quatrième lieu, une fois la légitimité du souverain garantie, et la subordination du gouvernement assurée par une constitution, la considération de la durée réapparaît dans le Contrat social, et elle contribue à modérer les textes emphatiques de Rousseau sur l'absence de contrainte pesant sur le souverain, et les thèses selon lesquelles le souverain serait toujours libre de réviser ses lois, même si elles sont bonnes. En réalité, libre de changer sa constitution, le peuple n'en doit rien faire et Rousseau montre que l'instabilité des lois est un mal politique.

Le refus de toute loi fondamentale qui pèserait légitimement sur la souveraineté populaire n'empêche donc pas qu'à l'instar de

27 Contrat social, III, 1, OC III, p. 396. 
Barbeyrac $^{28}$, Rousseau admette quelque chose qui aurait de la loi fondamentale la sagesse, la prudence et l'avantage de la durée. Par là il reconnaît une tension politique inévitable entre légitimité du pouvoir législatif et durée des $\operatorname{lois}^{29}$ :

Ce n'est point par les lois que l'Etat subsiste, c'est par le pouvoir législatif. La loi d'hier n'oblige pas aujourd'hui, mais le consentement tacite est présumé du silence, et le Souverain est censé confirmer incessamment les lois qu'il n'abroge pas, pouvant le faire. Tout ce qu'il a déclaré vouloir une fois, il le veut toujours, à moins qu'il ne le révoque. Pourquoi donc porte-t-on tant de respect aux anciennes lois ? C'est pour cela même. On doit croire qu'il n'y a que l'excellence des volontés antiques qui les ait pu conserver si longtemps; si le Souverain ne les eut reconnu constamment salutaires il les eut mille fois révoquées. ${ }^{30}$

\section{Les lois fondamentales de Pologne}

Le principe de légitimation de la loi par la coutume qui revient ici de façon étonnante, est strictement subordonné au principe de légitimation par la volonté générale. C'est sous cette seule condition que sont concédés certains arguments constitutionnalistes dans le Contrat social. Or les Lettres écrites de la Montagne et le texte sur la Pologne iront plus loin dans cette réhabilitation des lois fondamentales:

28 Barbeyrac, DNG, ibid., n (4), p. 292: «J'avoue, que si ces lois sont conformes au bien de la société, il ne doit pas les abolir légèrement tant que les choses demeurent dans la même situation. Mais ce n'est pas parce que ce sont des lois, qu'il s'est imposées, ou qu'il a établies au dessus de lui ; c'est uniquement en vertu de la loi naturelle, que le créateur et le législateur souverain impose à chaque société, aussi bien quà chaque personne, de prendre soin de sa propre conservation ».

29 Contrat social, II, XII, OC III, p. 393 : « Les lois qui règlent ce rapport portent le nom de lois politiques, et s'appellent aussi lois fondamentales, non sans quelque raison si ces lois sont sages. Car s'il n'y a dans chaque État qu'une bonne manière de l'ordonner, le peuple qui l'a trouvée doit s'y tenir: mais si l'ordre établi est mauvais, pourquoi prendrait-on pour fondamentales des lois qui l'empêchent d'être bon? D'ailleurs, en tout état de cause, un peuple est toujours le maître de changer ses lois, même les meilleures; car, s'il lui plait de se faire mal à lui-même, qui est-ce qui a droit de l'en empêcher? ». Ne retenir que l'un des éléments de cette tension, faire de Rousseau un pur souverainiste défenseur de la démocratie directe et absolue, ou un constitutionnaliste à la mode de Constant, c'est manquer le problème politique qu'il a affronté et vouloir le régler trop facilement.

30 Contrat social, III, 11 « De la mort du corps politique», p. 424. 
Dans les Etats où le gouvernement et les lois ont déjà leur assiette, on doit autant qu'il se peut éviter d'y toucher, et surtout dans les petites républiques, où le moindre ébranlement désunit tout. L'aversion des nouveautés est donc généralement bien fondée [...] quelque utiles que fussent des lois nouvelles, les avantages en sont presque toujours moins surs que les dangers n'en sont grands ${ }^{31}$.

Bref, l'Etat rousseauiste reçoit une constitution par défaut, celle qui détermine la forme du gouvernement, et sa stabilité reste toujours dépendante de la santé de la volonté générale. Ainsi en France les changements constitutionnels ne peuvent être pratiqués qu'avec des précautions particulières. C'est précisément sur cet effort de Rousseau pour mettre de côté certaines lois, qui ressemble bien à leur constitutionnalisation que nous nous arrêtons en cinquième et dernier lieu.

Rousseau reconnait que parmi les lois, certaines sont plus importantes que d'autres. Dans les Considérations sur le gouvernement de Pologne [1772], il réintroduit la possibilité de lois constitutionnelles, car il accepte une différence de statut entre les lois (alors que l'uniformité absolue de la loi s'y opposait dans le Contrat social). C'est par la modalité de révision spécifique que quelque chose comme une loi constitutionnelle peut être repéré dans sa pensée :

Il faut bien peser et bien méditer les points capitaux qu'on établira comme lois fondamentales et l'on fera porter sur ces points seulement la force du liberum veto. De cette manière on rendra la constitution solide et ces lois irrévocables autant qu'elles peuvent l'être : car il est contre la nature du corps politique de s'imposer des lois qu'il ne puisse révoquer ; mais il n'est ni contre la nature ni contre la raison qu'il ne puisse révoquer ces lois qu'avec la même solennité qu'il mit à les établir. Voilà toute la chaîne qu'il peut se donner à l'avenir. C'en est assez pour maintenir la constitution et pour contenter l'amour des Polonais pour le liberum veto sans s'exposer dans la suite aux abus qu'il a fait naître. ${ }^{32}$

31 Huitième des Lettres écrites de la Montagne, OC III, p. 946.

32 Considérations sur le gouvernement de Pologne, OC III, p. 996. 
De telles lois fondamentales doivent emporter l'unanimité. Or l'unanimité était l'apanage du contrat social ${ }^{33}$. De fait ces lois définies comme fondamentales engagent précisément la nature de ce corps politique fondé sur le contrat et Rousseau est bien ici en train d'accorder ainsi à certaines lois positives, la valeur fondatrice du contrat social lui-même ${ }^{34}$ : «Par le droit naturel des sociétés l'unanimité a été requise pour les lois fondamentales qui tiennent à son existence, telles par exemple, que la première corrigée, la cinquième et l'onzième [...] Or l'unanimité requise pour l'établissement de ces lois devra l'être de même pour leur abrogation ${ }^{35}$. Or en leur accordant une inviolabilité aussi haute que celle du contrat social, quelle meilleure garantie de stabilité peut-il leur donner ? L'idée de lois fondamentales entendues comme contraintes pesant sur le souverain a été écartée, mais elles reviennent ici comme constitution coessentielle au contrat social, lois relevant du même niveau légal que le contrat social, et contribuant pour ainsi dire à l'expliciter ${ }^{36}$.

Il est à remarquer à ce propos, qu'au moment de concéder des lois d'importance différente, Rousseau repense ses propres principes car ces lois fondamentales nous rappellent que les limites du pouvoir ne sont pas seulement artificielles, objet d'une volonté changeante, fût-elle

33 Contrat social, IV, 2, OC III, p. 439.

34 Ce point se distingue du texte de l'Emile cité plus haut et du point (2) de cet exposé où il n'était alors question d'accorder de valeur constitutionnelle quà un hypothétique contrat social.

35 Considérations sur le gouvernement de Pologne, OC III, p. 996. Ce texte est à rapprocher des occurrences de l'expression que I'on trouve dans le Discours sur l'économie politique, OC III, p. 243 : «Quoique les fonctions du père de famille et du premier magistrat doivent tendre au même but, c'est par des voies si différentes ; leurs devoirs et leurs droits sont si distingués qu'on ne peut les confondre sans se former de fausses idées des lois fondamentales de la société et sans tomber dans des erreurs fatales au genre humain. »; p. 256 : «Et il n'est pas plus croyable que la volonté générale consente qu'un membre de l'Etat quel qu'il soit en blesse ou en détruise un autre [...] car les conventions fondamentales étant enfreintes, on ne voit plus quel droit ni quel intérêt pourrait maintenir le peuple dans l'union sociale à moins qu'il n'y fût retenu par la seule force qui fait la dissolution de l'état civil ».

36 Létude de la portée du constitutionnalisme de Rousseau doit être complétée par celle des Lettres de la Montagne, et des articles de Céline Spector et Isabelle Bouvignies dans Religion, liberté, justice. Sur les Lettres écrites de la Montagne de J.J. Rousseau, dir. B. Bernardi, F. Guénard, G. Silvestrini, Paris, Vrin, 2005, respectivement : « Les principes de la liberté politique et la constitution d'Angleterre », p. 193-210, et «Constitutionnalisme et réformation dans les Lettres de la Montagne », p. 241-270.

Rev. Filos., Aurora, Curitiba, v. 28, n. 43, p. 19-38, jan./abr. 2016 
populaire, mais sont structurelles et tiennent surtout à la nature de la volonté générale. En effet, il est dit que les lois fondamentales tiennent au «droit naturel des sociétés » et renvoient aux limites internes du pouvoir souverain : celui-ci ne peut se retourner contre la fin pour laquelle il a été érigé et il ne peut notamment sacrifier un seul citoyen pour le salut de l'Etat. Le lien entre loi fondamentale et limite structurelle du pouvoir était déjà évoqué dans le Discours sur l'économie politique:

Et il n'est pas plus croyable que la volonté générale consente qu'un membre de l'Etat quel qu'il soit en blesse ou en détruise un autre [...] car les conventions fondamentales étant enfreintes, on ne voit plus quel droit ni quel intérêt pourrait maintenir le peuple dans l'union sociale à moins qu'il n'y fût retenu par la seule force qui fait la dissolution de l'état civil. ${ }^{37}$

La concession de ces lois fondamentales s'appuie donc sur la nature de la volonté générale, loin de l'idée d'une instance populaire désordonnée qui règnerait sur toutes les lois. Avant de devoir être rattachée à une tradition impersonnelle constitutionnaliste, on doit rattacher l'idée rousseauiste de limites du pouvoir à son analyse originale de la nature de la volonté générale, laquelle implique précisément des limites au pouvoir. Dans cette mesure, ce sont surtout les limites internes, essentielles à la nature du pouvoir politique, et non les limites externes, écrites qui comptent. Car de fait, la volonté générale n'est pas une instance incontrôlable, une volonté débridée, mais elle contient par sa nature même des contraintes que toute loi doit respecter.

$* * *$

Pour conclure, il y a bien un effort perceptible dans l'œuvre de Rousseau pour concilier constitutionnalisme et souveraineté populaire. Par la distinction du souverain et du gouvernement, par l'inaliénabilité

37 OC III, p. 256. 
de la souveraineté populaire, Rousseau est amené dans le Contrat social à penser la question des lois fondamentales, des bornes et limites $\mathrm{du}$ pouvoir souverain et donc des lois fondamentales d'une façon originale.

Pour résumer, il valorise dans les lois fondamentales d'un Etat l'ancienneté, la présomption de sagesse, mais sans engagement qui puisse menacer la souveraineté souveraineté. Plus fondamentalement, il rappelle l'existence de lois fondamentales des sociétés qu'on ne peut enfreindre et qui tiennent au droit naturel et à la raison d'être des sociétés politiques. Le travail conceptuel sur la notion de loi fondamentale effectué par Rousseau témoigne d'une tension interne à sa pensée entre le volontarisme, l'exigence d'une volonté générale toujours libre qui ne saurait se donner de chaînes et ne peut exister que pour le bien de chacun d'une part, et les exigences de la durée et de la continuité politiques d'autre part, qui réclament au contraire que la volonté puisse s'installer en habitude politique. La fonction d'encadrement du pouvoir politique que possèdent certaines lois ne concerne que le gouvernement et non pas le souverain, tandis que l'aspect coutumier, traditionnel et durable des lois fondamentales, qui était éclipsé par les jusnaturalistes, réapparaît chez Rousseau pour prêter sa force au contrat mais sans jamais primer seul ni valoir comme tel.

Ainsi, les tensions qui existent entre ces deux prétentions distinctes portées toutes deux par les lois fondamentales : l'ancienneté et la suprématie, montrent les difficultés que reconnaît Rousseau à concilier les fondements de droit des sociétés politiques avec leur histoire.

Dans ce cas, si la constitution et la notion de loi fondamentale posent de tels problèmes d'insertion dans la philosophie politique de Rousseau, on conçoit que la problématique des droits de l'homme poserait des problèmes similaires de conciliation de l'universel et du positif, du présent instantané et de la durée, de l'originel et de l'historique. Si donc Rousseau a inspiré le mouvement des droits de l'homme, c'est un problème, et non une solution, qu'il a légué. 


\section{Bibliographie}

AUDARD, C. Qu'est-ce que le libéralisme? Paris: Folio, 2009.

BOUVIGNIES, I. Constitutionnalisme et réformation dans les Lettres de la Montagne. In : Religion, liberté, justice. Sur les Lettres écrites de la Montagne de J.J. Rousseau. dir. B. Bernardi, F. Guénard, G. Silvestrini. Paris: Vrin, 2005. p. 241-270.

BURLAMAQUI, J-J. Principes du droit politique. Amsterdam : Châtelain, 1751. (reprint Caen, Centre de philosophie politique et juridique, 1983, 1. I, ch.7. Des caractères essentiels à la souveraineté, de ses modifications, de son étendue et de ses bornes, p. 60-88).

EHRARD, J. La notion de 'lois fondamentales' dans l'œuvre et la pensée de Montesquieu, Studies on Voltaire and the Eighteenth Century, 2005-5, p. 267-278.

HUNT, L. L'invention des droits de l'homme. Histoire, psychologie, politique. Trad. S. Kleiman-Lafon, Genève : Éd. Markus Haller, 2013.

KEOHANE, N. O. Philosophy and the State in France. The Renaissance to the Enlightenment. Princeton: Princeton University Press, 1980.

LEMAIRE, A. Les lois fondamentales de la monarchie française d'après les théoriciens de l'ancien régime. Paris: 1907. (reprint Genève, Slatkine-Megariotis, 1975)

PAINE, T. Les droits de l'homme. Paris, Belin : Éd. Claude Mouchard, 1987.

PUFENDORF, S. Droit de la Nature et des gens. Trad. Jean Barbeyrac, Bâle, 1732, Rééd. Caen, Centre de philosophie politique et juridique. Paris: Bibliothèque de philosophie politique et juridique, 1987.

ROUSSEAU, J-J. Euvres Complètes. Éd. Raymond et Gagnebin. Paris : La Pléiade-Gallimard. 5 vol. Tome III, 1964 (noté OC III).

SIEYES, E-J. Qu'est-ce que le tiers-état ? In : Écrits politiques. Éd. Roberto Zapperi. Paris: Gordon and Breach, 1994. 
SPECTOR, C. « Les principes de la liberté politique et la constitution d'Angleterre ». In : Religion, liberté, justice. Sur les Lettres écrites de la Montagne de J.J. Rousseau. dir. B. Bernardi, F. Guénard, G. Silvestrini. Paris : Vrin, 2005, p. 193-210.

SPITZ, J-F. John Locke et les fondements de la liberté moderne. Paris : Puf, 2001.

TERREL, J. Les théories du pacte social. Paris : Seuil, 2001.

Recebido: 20/10/2015

Received: 10/20/2015

Aprovado: 19/01/2016

Approved: 01/19/2016 